RUNHETC-2008-24

\title{
On Correlation Numbers in $2 D$ Minimal Gravity and Matrix Models
}

\author{
Talk presented at "Liouville Gravity and Statistical Models", International \\ conference in memory of Alexei Zamolodchikov, Moscow, June 21-24 2008
}

\author{
A.A.Belavin ${ }^{1}$, A.B. Zamolodchikov ${ }^{1,2}$ \\ ${ }^{1}$ L.D. Landau Institute for Theoretical Physics \\ Chernogolovka, 142432, Russia \\ ${ }^{2}$ NHETC, Department of Physics and Astronomy \\ Rutgers University \\ Piscataway, NJ 08855-0849, USA
}

\begin{abstract}
We test recent results for the four-point correlation numbers in Minimal Liouville Gravity against calculations in the oneMatrix Models, and find full agreement. In the process, we construct the resonance transformation which relates coupling parameters $\lambda_{k}$ of the Liouville Gravity with the couplings $t_{k}$ of the Matrix Models, up to the terms of the order 4. We also conjecture the general form of this transformation.
\end{abstract}

November 2008 


\section{Introduction}

At present, there are two relatively independent approaches to $2 D$ Quantum Gravity. One is the continuous approach, in which the theory is defined through the functional integral over the Riemannian 1 metric $g_{\mu \nu}(X)$, with appropriate gauge fixing. The choice of the conformal gauge leads to quantum Liouville theory [1] (coupled to matter fields), and for that reason this approach is often called the Liouville Gravity. The other is the discrete approach, based on the idea of approximating the fluctuating $2 D$ geometry by an ensemble of planar graphs, so that the continuous theory is recovered in the scaling limit where the planar graphs of very large size dominate. The discrete approach is usually referred to as the Matrix Models, since technically the ensemble of the graphs is usually generated by the perturbative expansion of the integral over $N \times N$ matrices, with $N$ sent to infinity to guarantee the planarity of the graphs (see e.g. [2] and references therein). Since these two approaches stem from the same idea of fluctuating geometry, they are expected to produce identical results for the physical quantities. Indeed, this expectation was confirmed by explicit calculations of some quantities in a number of particular models [3], [4], [5]. However, the techniques involved in calculations by the two approaches are very different, and satisfactory conceptual understanding of the relation between those techniques is still lacking. In the absence of that, more checks of the agreement of the results seem to be desirable.

The most easily-defined objects in the Quantum Gravity are the n-point "correlation numbers" - the integrated correlation functions

$$
C_{k_{1} \ldots k_{n}}=\left\langle O_{k_{1}} \ldots O_{k_{n}}\right\rangle, \quad O_{k}=\int_{\mathbb{M}} \mathcal{O}_{k}(X)
$$

Here $\mathcal{O}(X)$ are some local densities (two forms) on the manifold $\mathcal{M}$ which may involve both the "matter" and the metric degrees of freedom localized at $X \in \mathbb{M}$, and the expectation value is taken over the fluctuations of both the matter and the geometry. The generating function

$$
Z\left(\left\{\lambda_{k}\right\}\right)=Z_{0}\left\langle\exp \left\{\sum_{k} \lambda_{k} O_{k}\right\}\right\rangle
$$

may be regarded as the vacuum partition function of the original theory perturbed by adding the fields $\mathcal{O}_{k}(X)$ to the action density, with the coupling constants $-\lambda_{k}$. Throughout this paper we limit our attention to the case when $\mathbb{M}$ is topologically a sphere. Even in this case, while it is relatively easy to evaluate the $n$-point correlation numbers in solvable Matrix

\footnotetext{
${ }^{1}$ Here we always have in mind the Euclidean version of the theory.
} 
Models, the Liouville Gravity approach to these quantities involves technically complicated integration over the $n-3$ dimensional moduli space of a sphere with $n$ punctures. For this reason, most previous comparisons of the Matrix Models and the Liouville Gravity results was limited to one-, two-, and three-point correlation numbers (Notable exceptions are the analysis in [5]). A few years ago, a new technique for handling the moduli integrals was developed by Alexei Zamolodchikov and one of the authors [6]. It applies to the so-called "Minimal Gravity" models (minimal CFT coupled to the Liouville mode); by using the Higher Liouville Equations of motion [7] it allows one to reduce the moduli integrals to the boundary terms. In this way the (partial) result for the four-point correlation numbers in the Minimal Gravity was obtained in [6].

In this note we test the result of [6] against the corresponding correlation numbers from the Matrix Models. There is an important subtlety which makes this comparison less than straightforward. In a local field theory, the integrated correlation functions of the type (1.1) suffer from intrinsic ambiguity generally referred to as the "contact terms". Since (1.1) is the integral

$$
\int_{X_{1}, \ldots, X_{n}}\left\langle\mathcal{O}_{k_{1}}\left(X_{1}\right) \ldots \mathcal{O}_{k_{n}}\left(X_{n}\right)\right\rangle,
$$

the correlation numbers may pick up contributions from delta-like terms in the integrand, when two or more points $X_{i}$ collide. Although most of the contact terms are not determined by the field theory itself, it is well known that any change of contact terms is equivalent to a certain analytic change of the coupling parameters in (1.2),

$$
\lambda_{k} \rightarrow \tilde{\lambda}_{k}=\lambda_{k}+\sum_{k_{1} k_{2}} C_{k}^{k_{1} k_{2}} \lambda_{k_{1}} \lambda_{k_{2}}+\cdots
$$

Of course, this relation is at the heart of the renormalization theory, and indeed the ambiguity of the contact terms just reflects the freedom of making finite renormalizations. Usually, the ambiguity is resolved by appealing to the dimensional analysis. The local fields $\mathcal{O}_{k}(X)$ can be chosen in such a way that they (and hence the associated coupling parameters in (1.2)) have definite mass dimensions, so that

$$
O_{k} \sim\left[\operatorname{mass}^{2}\right]^{\delta_{k}}, \quad \lambda_{k} \sim\left[\operatorname{mass}^{2}\right]^{-\delta_{k}}
$$

Then, if one does not allow for any "auxiliary" dimensional parameters, only transformations (1.4) which respect the balance of the dimensions are admitted, i.e. the non-linear (say, $n$-th 
order) terms in (1.4) are admissible only if they satisfy the "resonance conditions"?

$$
\delta_{k}=\delta_{k_{1}}+\ldots+\delta_{k_{n}}
$$

We generally refer to the non-linear terms in (1.4) as the "resonance terms".

In the absence of the resonances, no nonlinear finite renormalizations (1.4) consistent with the dimensional counting are allowed, and thus the above ambiguity is completely fixed. However, when the Minimal CFT is coupled to the gravity, appearance of the resonances turns out to be very common. Therefore this ambiguity needs to be resolved before a meaningful comparison between the correlation numbers obtained by the two different methods can be made. The problem was first outlined by Moore, Seiberg, and Staudacher [8], who also found the solution in a number of special cases. In particular, addressing the case of the $p$-critical point of the one-Matrix Model and the corresponding Minimal Gravity $\mathcal{M G}_{2 / 2 p+1}$ (the Minimal CFT $\mathcal{M}_{2 / 2 p+1}$ coupled to the Liouville Gravity), they partly determined the resonance terms in the relation between the coupling parameters, which allowed them to establish equivalence up to the level of two-point correlation numbers.

In what follows we extend this analysis to three- and four-point correlation numbers, and find perfect agreement between the Matrix Models and Minimal Gravity results. In the process, we determine the higher order resonance terms by demanding that the higher order correlation numbers satisfy the fusion rules inherent to the Minimal Gravity. At the end, we conjecture the full resonance transformation which relates coupling parameters in the $p$-critical one-Matrix Models and Minimal Gravity $\mathcal{M G}_{2 / 2 p+1}$.

\section{Minimal Gravity $\mathcal{M G}_{2 / 2 p+1}$}

Specific models of the Liouville Gravity are defined by the content of the "matter" field theory which is placed on the $2 D$ manifold with the fluctuating metric. Perhaps the simplest models are defined by choosing the Minimal $\mathrm{CFT} \mathcal{M}_{q^{\prime} / q}$ as the "matter" theory. As in [9], we use the term "Minimal Gravity" $\mathcal{M G}_{q^{\prime} / q}$ for such models. In this work we restrict attention to the models with $q^{\prime}=2$ and $q=2 p+1, p=1,2,3, \ldots$ The Minimal Gravity $\mathcal{M G}_{2 / 2 p+1}$ is very likely to correspond to the $p$-critical point in the general one-Matrix Model [10].

\footnotetext{
${ }^{2}$ The term is taken from the vocabulary of the Renormalization Group, where precisely these conditions characterize the nonlinear terms in the beta-functions which can not be eliminated by analytic transformations of the coupling parameters.
} 


\subsection{Minimal Model $\mathcal{M}_{2 / 2 p+1}$}

The Kac table of the Minimal CFT $\mathcal{M}_{2 / 2 p+1}$ is a single raw of the length $2 p$, the entries being the degenerate primary fields $\Phi_{(1, n)}, n=1,2, \ldots, 2 p$. Due to the mandatory identification $\Phi_{(1, n)}=\Phi_{(1,2 p+1-n)}$, the model actually has only $p$ independent primary fields. We will use the abbreviated notation

$$
\Phi_{k}=\Phi_{(1, k+1)}
$$

for them, so that $\Phi_{0}$ is the identity operator. All the independent primaries are listed by letting $k$ run through the range $k=0,1,2, \ldots, p-1$ (and in what follows, unless stated otherwise, it is assumed that $k$ lies in this range), but it is often convenient to extend the range to the full Kac table, $k=1,2, \ldots, 2 p$, with the above identification

$$
\Phi_{2 p-k-1}=\Phi_{k}
$$

kept in mind. In particular, with this convention the fusion rules of $\mathcal{M}_{2 / 2 p+1}$ take the simple form

$$
\left[\Phi_{k_{1}}\right]\left[\Phi_{k_{2}}\right]=\sum_{k=\left|k_{1}-k_{2}\right|: 2}^{k_{1}+k_{2}}\left[\Phi_{k}\right]
$$

where as usual $\left[\Phi_{k}\right]$ stands for the irreducible Virasoro representation associated with the primary field $\Phi_{k}$. Here and below the symbol $\sum_{k=m: 2}^{m^{\prime}}$ denotes the sum with the step 2, in which $k$ runs over the values $m, m+2, m+4, \ldots, \leq m^{\prime}$. While in (2.3) it is assumed that $k_{1}, k_{2}$ lie within the domain $[0,1, \ldots, p-1]$, the summation index $k$ is allowed to run outside it, where the terms $\left[\Phi_{k}\right]$ are understood as $\left[\Phi_{2 p-k-1}\right]$.

Note that the identification (2.2) breaks the naive parity symmetry $\Phi_{k} \rightarrow(-)^{k} \Phi_{k}$ of $(2.3)$, so that $\left[\Phi_{k}\right]$ with odd (even) $k$ may appear in the r.h.s of (2.3) with even (odd) $k_{1}+k_{2}$. In particular, the correlation functions

$$
\left\langle\Phi_{k_{1}}\left(X_{1}\right) \Phi_{k_{2}}\left(X_{2}\right) \ldots \Phi_{k_{n}}\left(X_{n}\right)\right\rangle
$$

do not necessarily vanish when $k_{1}+k_{2}+\ldots+k_{n}$ is odd.

On the other hand, conformal invariance and the fusion rules (2.3) do force many correlation function to vanish. Thus, the conformal invariance demands vanishing of all the one-point correlation function except for $\left\langle\Phi_{0}(X)\right\rangle$, as well as the diagonal form of the twopoint functions, i.e.

$$
\begin{array}{lll}
\left\langle\Phi_{k}(X)\right\rangle=0 & \text { unless } & k=0, \\
\left\langle\Phi_{k_{1}}\left(X_{1}\right) \Phi_{k_{2}}\left(X_{2}\right)\right\rangle=0 & \text { unless } & k_{1}=k_{2},
\end{array}
$$


while the fusion rules (2.3) impose restrictions on the multi-point correlation functions (2.4) with $n \geq 3$. Those restriction can be written in a compact form

$$
\text { (2.4) }=0 \text { if }\left\{\begin{array}{clll}
k_{1}+\ldots+k_{n-1}<k_{n} & \text { for } & k_{1}+\ldots+k_{n} \text { even } \\
k_{1}+\ldots+k_{n}<2 p-1 & \text { for } & k_{1}+\ldots+k_{n} & \text { odd }
\end{array}\right.
$$

if one assumes that all $k_{i}$ are in the range $[0,1, \ldots, p-1]$, and that $k_{n}$ is the largest of them, i.e. $k_{i} \leq k_{n}$. Below we generally refer to the case of even or odd $k_{1}+\ldots+k_{n}$ as the even and odd sectors.

\subsection{Coupling to Liouville Gravity}

According to [1] [11], coupling a conformally invariant "matter" field theory to the fluctuating metric $g_{\mu \nu}(x)$ leads, in the conformal gauge, to the famous Liouville action

$$
\mathcal{A}_{L}=\frac{1}{4 \pi} \int_{\mathbb{M}} \sqrt{\hat{g}}\left[\hat{g}^{\mu \nu} \partial_{\mu} \varphi \partial_{\nu} \varphi+Q \hat{R} \varphi+4 \pi \mu e^{2 b \varphi}\right] d^{2} x
$$

for the field $\varphi$ related to the conformal factor in $g_{\mu \nu}=e^{2 b \varphi} \hat{g}_{\mu \nu}$, where $\hat{g}_{\mu \nu}$ is an arbitrary fixed "background" metric on $\mathbb{M}$. The parameters $b$ and $Q$ are determined by the central charge $c_{M}$ of the conformal "matter",

$$
26-c_{M}=1+6 Q^{2} \quad Q=1 / b+b .
$$

In our case the "matter" is the Minimal CFT $\mathcal{M}_{2 / 2 p+1}$ with $c_{M}=-6 p+10-\frac{12}{2 p+1}$, i.e.

$$
b=\sqrt{\frac{2}{2 p+1}} .
$$

The parameter $\mu$ in (2.8) is dimensional,

$$
\mu \sim[\operatorname{mass}]^{2},
$$

and interpreted as the cosmological constant.

The operators $O_{k}=\int_{\mathbb{M}} \mathcal{O}_{k}(x)$ are constructed by "gravitational dressing" of the primary fields $\Phi_{k}$,

$$
\mathcal{O}_{k}(x)=\Phi_{k}(x) e^{2 a_{k} \varphi(x)} \sqrt{\hat{g}} d^{2} x,
$$


where $a_{k}=b \frac{k+2}{2}$. This choice of the parameters $a_{k}$ ensures that the integrand in (2.12) is indeed a density. These parameters also determine the mass dimensions of the operators $O_{k}$, the $\delta_{k}$ in (1.5) [3] [11],

$$
\delta_{k}=-\frac{a_{k}}{b}=-\frac{k+2}{2}
$$

Proper gauge fixing leads to the following expression for the correlation numbers (1.1) with $n \geq 3$

$$
\left\langle O_{k_{1}} \ldots O_{k_{n}}\right\rangle=\int_{x_{1}, \ldots, x_{n-3}}\left\langle\mathcal{O}_{k_{1}}\left(x_{1}\right) \ldots \mathcal{O}_{k_{n-3}}\left(x_{n-3}\right) \tilde{\mathcal{O}}_{k_{n-2}}\left(x_{n-2}\right) \tilde{\mathcal{O}}_{k_{n-1}}\left(x_{n-1}\right) \tilde{\mathcal{O}}_{k_{n}}\left(x_{n}\right)\right\rangle_{\substack{\text { Matter \& } \\ \text { Liouville }}},
$$

where $\tilde{\mathcal{O}}_{k}=C \bar{C} e^{2 a_{k} \varphi} \Phi_{k}$ are scalars (zero forms) associated with the densities $\mathcal{O}_{k}$. Three points $x_{n-2}, x_{n-1}, x_{n}$ can be chosen at will, and the integration is performed over the remaining $n-3$ points $x_{1}, \ldots, x_{n-3}$ (interpreted as the moduli of the $n$-punctured sphere). The expectation value at the right hand side of (2.14) involves both the "matter" and the Liouville sectors. Due to the factorized form (2.12), the integrand in (2.14) is a product of the correlation functions (2.4) and the correlation functions of the Liouville exponentials

$$
\left\langle e^{2 a_{k_{1}} \varphi\left(x_{1}\right)} \ldots e^{2 a_{k_{n}} \varphi\left(x_{n}\right)}\right\rangle_{\text {Liouville }}
$$

For $n=3$ no integration is necessary, and the three-point correlation numbers are obtained by multiplying the three-point functions (2.4) by the Liouville three-point function (2.15). The result takes a very simple form [9]

$$
\left\langle O_{k_{1}} O_{k_{2}} O_{k_{3}}\right\rangle=-\mu^{\delta_{k_{1}}+\delta_{k_{2}}+\delta_{k_{3}}} N_{k_{1} k_{2} k_{3}} \mathcal{N}_{p} \prod_{i=1}^{3} \operatorname{Leg}_{L}\left(k_{i}\right)
$$

Here

$$
\mathcal{N}_{p}=(2 p-1)(2 p+1)(2 p+3)
$$

and the "leg factors"

$$
\operatorname{Leg}_{L}(k)=\frac{(-)^{\frac{k}{2}}}{2 \pi^{\frac{k}{2}}}\left[\gamma\left(\frac{2}{2 p+1}\right)\right]^{-\frac{k+1}{2}}\left[\gamma\left(\frac{2(k+1)}{2 p+1}\right)\right]^{\frac{1}{2}} \frac{\Gamma(p-1 / 2)}{\Gamma(p-k-1 / 2)}
$$


$(\gamma(t)=\Gamma(t) / \Gamma(1-t))$ will play a very small role in our discussion 3 . One can always get rid of them by multiplicative renormalization of the operators $O_{k}$ and the coupling parameters $\lambda_{k}$ in (1.2)

$$
O_{k} \rightarrow \frac{1}{\operatorname{Leg}_{L}(k)} O_{k}, \quad \lambda_{k} \rightarrow \operatorname{Leg}_{L}(k) \lambda_{k}
$$

The most important entry in (2.16) is the "fusion coefficients" $N_{k_{1} k_{2} k_{3}}$ which take the value 1 if three-point fusion rules are satisfied, and turns to zero otherwise. Explicitly,

$$
N_{k_{1} k_{2} k_{3}}=\left\{\begin{array}{lc}
1 \text { if } & k_{1}+k_{2}+k_{3} \geq 2 p-1 \\
0 & \text { otherwise }
\end{array} \text { for } k_{1}+k_{2}+k_{3}\right. \text { odd }
$$

and

$$
N_{k_{1} k_{2} k_{3}}=\left\{\begin{array}{lr}
1 \text { if } & k_{1}+k_{2} \geq k_{3} \\
0 & \text { otherwise }
\end{array} \quad \text { for } \quad k_{1}+k_{2}+k_{3}\right. \text { even }
$$

where in writing (2.21) we have assumed that $k_{3}$ is the maximal of the numbers $k_{i}$, i.e. $k_{1}, k_{2} \leq k_{3}$. This factor of course is inherited from the "matter" three-point functions $\left\langle\Phi_{k_{1}} \Phi_{k_{2}} \Phi_{k_{3}}\right\rangle$.

Since $\Phi_{0}$ is the identity operator in $\mathcal{M}_{2 / 2 p+1}$, insertion of $O_{0}$ in (1.1) is equivalent to taking the derivative with respect to $\mu$, more precisely

$$
\left\langle O_{0} O_{k_{1}} \ldots O_{k_{n}}\right\rangle=-Z_{0}^{-1} \frac{\partial}{\partial \mu}\left(Z_{0}\left\langle O_{k_{1}} \ldots O_{k_{n}}\right\rangle\right)
$$

where

$$
Z_{0} \sim \mu^{\frac{2 p+3}{2}}
$$

is the Liouville partition function of a sphere. Therefore the two- and one-point correlation numbers are easily deduced from (2.16),

$$
\left\langle O_{k} O_{k^{\prime}}\right\rangle=\delta_{k, k^{\prime}} \frac{\mathcal{N}_{p} \mu^{2 \delta_{k}}}{2 p-2 k-1} \operatorname{Leg}_{L}^{2}(k), \quad\left\langle O_{k}\right\rangle=-\delta_{k, 0}(p+3 / 2) \mu^{-1}
$$

\footnotetext{
${ }^{3}$ Note that $\operatorname{Leg}_{L}(0)=1 / 2$. It is also interesting to note that for odd $k$ the factor (2.18) returns pure imaginary values. This reflects the known property of the odd- $k$ operators $\Phi_{k}$ in the Minimal CFT $\mathcal{M}_{2 / 2 p+1}$. For example, $\mathcal{M}_{2 / 5}$ is known to describe the Yang-Lee edge criticality [12]; in this identification the operator $\Phi_{1}$ corresponds to the Ising order parameter, which couples to the pure imaginary magnetic field.
} 
Again, the diagonal form of the two-point numbers and vanishing of all but one of the onepoint numbers can be traced to the corresponding properties of the "matter" correlation functions, Eq's (2.6) and (2.5).

In Ref. [6] the integration over the moduli in (2.14) is performed in the case $n=4$. The result, specialized for $\mathcal{M G}_{2 / 2 p+1}$, can be written as

$$
\left\langle O_{k_{1}} O_{k_{2}} O_{k_{3}} O_{k_{4}}\right\rangle=\mathcal{N}_{p} \mu^{\sum \delta_{k_{i}}} C_{k_{1} k_{2} k_{3} k_{4}} \prod_{i=1}^{4} \operatorname{Leg}_{L}\left(k_{i}\right)
$$

where, if one chooses $k_{1}$ to be the smallest of the numbers $k_{i}$ (i.e. $k_{1} \leq k_{2}, k_{3}, k_{4}$ ), the factor $C_{k_{1} k_{2} k_{3} k_{4}}$ has the form

$$
C_{k_{1} k_{2} k_{3} k_{4}}=\left(k_{1}+1\right)\left(p+k_{1}+3 / 2\right)-\sum_{i=2}^{4} \sum_{s=-k_{1}: 2}^{k_{1}}\left|p-k_{i}-s-\frac{1}{2}\right| .
$$

An important qualification applies to this result. Eq.(2.26) was obtained under the assumption that the number of conformal blocks in the decomposition of the $\mathcal{M}_{2 / 2 p+1}$ correlation function $\left\langle\Phi_{k_{1}} \Phi_{k_{2}} \Phi_{k_{3}} \Phi_{k_{4}}\right\rangle$ is exactly $k_{1}$. To put it in explicit form, assume that $k_{i}$ are arranged in non-increasing order,

$$
k_{1} \leq k_{2} \leq k_{3} \leq k_{4} \leq p-1
$$

Then, in the even sector, this assumption is fulfilled iff

$$
k_{1}+k_{4} \leq k_{2}+k_{3}
$$

(which incidentally guarantees that the even-sector fusion rules are satisfied). In the odd sector this condition also requires that the fusion rules are satisfied with sufficient redundancy

$$
-k_{1}+k_{2}+k_{3}+k_{4} \geq 2 p-1
$$

which in turn demands validity of (2.28) ( since $k_{4} \leq p-1$ ). Thus, in both even and odd sectors, when (2.28) breaks down, the validity of (2.26) is questionable [6]. Indeed, we will see in Section 4.2 that at $k_{1}+k_{4}>k_{2}+k_{3}(2.26)$ deviates from the Matrix Model result.

As was mentioned in the Introduction, the Minimal Gravity $\mathcal{M G}_{2 / 2 p+1}$ is likely to be the world-sheet theory of the $p$-critical point in the one-Matrix Model [10]. This identification was confirmed by explicit comparison of the one- and two-point correlation numbers [8]. The aim of this paper is to extend the analysis to the higher order correlation numbers, and in particular to test the new result (2.26) against the Matrix Models calculations. 


\section{One-Matrix Model}

The matrix models technique was extensively studied in the literature. Most of what we will need here can be found in Ref. [2] and references therein.

\section{$3.1 \quad p$-critical point}

The one-Matrix Model exhibits an infinite set of multi-critical points, labelled by the integer $p=1,2,3, \ldots$. In the scaling limit near the $p$-critical point, the partition function of the sphere is expressed through the solution of the "string equation"

$$
\mathcal{P}(u)=0,
$$

where $\mathcal{P}(u)$ is the $p+1$-degree polynomial

$$
\mathcal{P}(u)=u^{p+1}+t_{0} u^{p-1}+\sum_{k=1}^{p-1} t_{k} u^{p-k-1}
$$

with the parameters $t_{k}$ describing the relevant deviations from the $p$-critical point 4 . The singular part of the Matrix Model partition function $Z\left(t_{0}, t_{1}, \ldots, t_{p-1}\right)$ is expressed through (3.2) as follows,

$$
Z=\frac{1}{2} \int_{0}^{u_{*}} \mathcal{P}^{2}(u) d u
$$

where $u_{*}=u_{*}\left(t_{0}, t_{1}, \ldots, t_{p-1}\right)$ is the suitably chosen root of the polynomial (3.2), i.e. $\mathcal{P}\left(u_{*}\right)=$ 0 . It is important to remember that (3.3) really gives only the singular part of the Matrix Model partition function. The actual matrix integral has also a regular part, analytic in all the parameters $t_{k}$ at $\left\{t_{k}\right\}=0$; in the spirit of the scaling theory of criticality, the regular part is disregarded as non-universal. For this reason the choice of 0 as the lower limit in the integral (3.3) is largely arbitrary. One can replace it by any constant, or indeed any regular function of $t_{k}$, at the price of adding the regular terms to $Z$.

There is strong evidence [10], [8] that (3.3) (with $u_{*}$ taken to be the maximal real root of $\mathcal{P}(u)$ ) provides the Matrix Model description of the Minimal Gravity $\mathcal{M G}_{2 / 2 p+1}$, perturbed by the operators (2.12). In this identification $-t_{0}$ is interpreted as the cosmological constant

\footnotetext{
${ }^{4}$ Note that our labelling of the parameters $t_{k}$ is different from that in Ref. [8]; our $t_{k}$ are $t_{p-k-1}$ in [8].

${ }^{5}$ In the Matrix Models technique it actually emerges through the equation $\partial^{2} Z / \partial t_{p-1}^{2}=u_{*}\left(t_{0}, \ldots, t_{p-1}\right)$. The Eq.(3.3) gives "physical" solution of this equation.
} 
$\mu$ in (2.8). Obvious re-scaling symmetry $u \rightarrow a u, t_{k} \rightarrow a^{k+2} t_{k}$ of Eq.(3.1) allows one to ascribe "mass dimensions" to the the parameters $t_{k}$,

$$
t_{k} \sim\left[\operatorname{mass}^{2}\right]^{\frac{k+2}{2}}
$$

and then $Z \sim\left[\mathrm{mass}^{2}\right]^{\frac{2 p+3}{2}}$, in agreement with (2.13) and (2.23).

Comparison at the level of the correlation numbers involves two subtleties noticed long ago in Ref. [8]. First, it is obvious from (2.13) that at sufficiently large $p$ there are many resonances (1.6) between the dimensions $\delta_{k}$. Therefore the relation between the parameters $t_{k}$ in (3.1) and the Minimal Gravity couplings $\lambda_{k}$ may involve the resonance terms

$$
t_{k}=C_{k} \lambda_{k}+\sum_{n=2}^{\left[\frac{k+2}{2}\right]} \sum_{k_{1}, \ldots, k_{n}=0}^{p-1} C_{k}^{k_{1} \ldots k_{n}} \lambda_{k_{1}} \ldots \lambda_{k_{n}}
$$

with the coefficients constrained by the condition

$$
C_{k}^{k_{1} \ldots k_{n}}=0 \quad \text { unless } \quad \sum_{i=1}^{n} k_{i}=k+2-2 n .
$$

The sum over $k_{i}=0,1, \ldots, p-1$ in (3.5) includes $k_{i}=0$ to take into account the possibility that the integer powers of the cosmological constant $\mu$ appear in the right-hand side; by definition

$$
\lambda_{0}=-\mu \text {. }
$$

The coefficients $C_{k}$ have very little significance. They can be removed by trivial renormalizations of the parameters $\lambda_{k}$ (or $t_{k}$ ) similar to (2.19). Physical equivalence between the $p$-critical Matrix Model and the Minimal Gravity $\mathcal{M G}_{2 / 2 p+1}$ would imply that by special choice of the coefficients in $C_{k}^{k_{1} \ldots k_{n}}$ in (3.5) the partition function (3.3), expressed through $\left\{\lambda_{k}\right\}$, can be made identical to the generating function (1.2) of the Minimal Gravity, up to regular terms. This was the idea put forward by Moore, Seiberg, and Staudacher [8], who have verified the identity up to the two-point correlation numbers. In the next subsection we warm up by re-deriving their result in somewhat different language, and then proceed to the analysis of the higher orders.

The quantities to be compared with the correlation numbers of $\mathcal{M G}_{2 / 2 p+1}$ are the coefficients of the expansion

$$
Z=Z_{0}+\sum_{k=1}^{p-1} \lambda_{k} Z_{k}+\sum_{k_{1} k_{2}=1}^{p-1} \frac{\lambda_{k_{1}} \lambda_{k_{2}}}{2} Z_{k_{1} k_{2}}+\ldots+\sum_{k_{1}, \ldots k_{n}=1}^{p-1} \frac{\lambda_{k_{1}} \ldots \lambda_{k_{n}}}{n !} Z_{k_{1} \ldots k_{n}}+\ldots
$$


of the Matrix Model partition function (3.3) in the powers of $\lambda_{1}, \ldots \lambda_{p-1}$, with $\lambda_{0}=-\mu$ kept fixed. By dimensional analysis

$$
Z_{k_{1} \ldots k_{n}}=z_{k_{1} \ldots k_{n}} \mu^{\frac{2 p+3-2 n-\sum k_{i}}{2}}
$$

with $z_{k_{1} \ldots k_{n}}$ being just numbers. The coefficients with even $\sum_{i=1}^{n} k_{i}$ (even sector) are halfinteger powers of $\mu$, and thus definitely belong to the singular part of the partition function. However, $Z_{k_{1} \ldots k_{n}}$ with odd $\sum_{i=1}^{n} k_{i}$ (odd sector) involve integer powers of $\mu$. When also

$$
\sum_{i=1}^{n} k_{i} \leq 2 p+3-2 n
$$

the odd-sector coefficients are non-negative powers of $\mu$, and thus belong to the regular part of the partition function. As such, they can be adjusted at will, and will be of no interest in our analysis 6. Note that this inequality is always satisfied for $n=1,2$, but at $n \geq 3$ negative powers of $\mu$ appear. Therefore it is meaningful to compare the odd-sector correlation numbers with $\sum k_{i}>2 p+3-2 n$ with the results in $\mathcal{M G}_{2 / 2 p+1}$. We will return to this point in Sections 4 and 5 below.

\subsection{One- and two-point correlation numbers}

When one plugs (3.5) into (3.2), the polynomial takes the form

$$
\mathcal{P}(u)=\mathcal{P}_{0}(u)+\sum_{k=1}^{p-1} \lambda_{k} \mathcal{P}_{k}(u)+\ldots+\sum_{k_{i}=1}^{p-1} \frac{\lambda_{k_{1}} \ldots \lambda_{k_{n}}}{n !} \mathcal{P}_{k_{1} \ldots k_{n}}(u)+\ldots
$$

where $\mathcal{P}_{0}(u)$ and $\mathcal{P}_{k_{1} \ldots k_{n}}(u)$ are the polynomials whose coefficients involve non-negative powers of $\mu$. By dimensional analysis

$$
\begin{aligned}
& \mathcal{P}_{0}(u)=u^{p+1}+C_{0}^{\prime} \mu u^{p-1}+C_{0}^{\prime \prime} \mu^{2} u^{p-3}+\ldots \\
& \mathcal{P}_{k}(u)=C_{k} u^{p-k-1}+C_{k}^{\prime} \mu u^{p-k-3}+C_{k}^{\prime \prime} \mu^{2} u^{p-k-5}+\ldots \\
& \ldots
\end{aligned}
$$

\footnotetext{
${ }^{6}$ However, note that these regular terms are "special", in that they fully agree with the scaling (the coefficients $z_{k_{1} \ldots k_{n}}$ in (3.9) are dimensionless). Whereas generic regular terms of the full microscopic partition function violate the scaling, and thus are definitely beyond control of continuous field theory, there is much reason to think that in Quantum Gravity the special regular terms can be attributed some universal meaning (see [13]). In this work we do not address this interesting line of questions.
} 
(here $C_{k}$ are the same as in (3.5), and $C_{k}^{\prime}, C_{k}^{\prime \prime}, \ldots$ are dimensionless constants related to the higher-order coefficients in (3.5)), and in general $\mathcal{P}_{k_{1} \ldots k_{n}}(u)$ are polynomials of the degree

$$
p+1-2 n-\sum k_{i},
$$

of similar structure. Of course, only polynomials of non-negative degree appear, so that the sum in (3.11) is finite.

It is essential to notice that all the polynomials are either even or odd,

$$
\mathcal{P}_{k_{1} \ldots k_{n}}(-u)=(-)^{p+1-\sum k_{i}} \mathcal{P}_{k_{1} \ldots k_{n}}(u),
$$

because only integer powers of $\mu$ can appear in (3.12). We use this symmetry to rewrite Eq.(3.3) in a somewhat different form, more convenient for our analysis below. We split the integration domain into two pieces

$$
\int_{0}^{u_{*}}=\int_{u_{0}}^{u_{*}}+\int_{0}^{u_{0}}
$$

where $u_{0}$ stands for the root of $\mathcal{P}_{0}(u)$ associated with $u_{*}$, i.e. $u_{*}$ at $\lambda_{1}, \ldots, \lambda_{p-1}=0$. Note that

$$
u_{0}=a_{0} \mu^{\frac{1}{2}}
$$

with some constant $a_{0}$ which plays no significant role in our discussion below. The integrand in (3.3) involves the products

$$
\mathcal{P}_{k_{1} \ldots k_{m}}(u) \mathcal{P}_{k_{m+1} \ldots k_{n}}(u)
$$

which are even or odd in $u$ depending on whether $k_{1}+\ldots+k_{n}$ is even or odd. For the even terms we can extend the integration in the second piece in (3.15) to the domain $\left[-u_{0}, u_{0}\right]$,

$$
\int_{0}^{u_{0}} \rightarrow \frac{1}{2} \int_{-u_{0}}^{u_{0}}
$$

On the other hand, it is not difficult to see that the contributions of the odd terms in that piece involve only non-negative integer powers of $\mu$, and thus belong to the regular part of the partition function. Thus, up to regular terms

$$
Z=\frac{1}{2} \int_{u_{0}}^{u_{*}} \mathcal{P}^{2}(u) d u+\frac{1}{4} \int_{-u_{0}}^{u_{0}} \mathcal{P}^{2}(u) d u,
$$


and since the regular terms are of no interest to us here, in what follows we study the partition function (3.18).

The first order of business is to determine $\mathcal{P}_{0}(u)$ and $\mathcal{P}_{k}(u)$. Since $u_{*}=u_{0}+O\left(\lambda_{k}\right)$, the first term in (3.18) does not contribute to the first three orders in the expansion (3.8), and one finds

$$
\begin{aligned}
& Z_{0}=\frac{1}{4} \int_{-u_{0}}^{u_{0}} \mathcal{P}_{0}^{2}(u) d u \\
& Z_{k}=\frac{1}{2} \int_{-u_{0}}^{u_{0}} \mathcal{P}_{0}(u) \mathcal{P}_{k}(u) d u \\
& Z_{k_{1} k_{2}}=\frac{1}{2} \int_{-u_{0}}^{u_{0}}\left[\mathcal{P}_{k_{1}}(u) \mathcal{P}_{k_{2}}(u)+\mathcal{P}_{0}(u) \mathcal{P}_{k_{1} k_{2}}(u)\right] d u
\end{aligned}
$$

Agreement with (2.24) requires that all $Z_{k}$ vanish. Then (3.20) suggests that all the polynomials $\mathcal{P}_{k}(u)$ must be orthogonal to $\mathcal{P}_{0}(u)$ on the interval $\left[-u_{0}, u_{0}\right]$, with the measure 1. Since the degrees of all $\mathcal{P}_{k_{1} k_{2}}(u)$ are smaller than $p-2$ (indeed, smaller than $p-4$ ), the second term in (3.21) may be disregarded, and then agreement with the diagonal form of the two-point correlation numbers in (2.24) requires that $\mathcal{P}_{k}(u)$ themselves form an orthogonal set of polynomials on that interval. One concludes that $\mathcal{P}_{k}(u)$, up to normalization, are the Legendre polynomials,

$$
\mathcal{P}_{k}(u)=C_{k} g_{k} u_{0}^{p-k-1} P_{p-k-1}\left(u / u_{0}\right) .
$$

Here $C_{k}$ are the same as in (3.12), and

$$
g_{k}=\frac{(p-k-1) !}{(2 p-2 k-3) ! !}
$$

We review some trivia about the Legendre polynomials in the Appendix A (see e.g. [14] for systematic display). Furthermore, since $\mathcal{P}_{0}(u)$ is $p+1$ degree polynomial, with no $u^{p}$ term, and vanishing at $u_{0}$, one finds

$$
\mathcal{P}_{0}(u)=g u_{0}^{p+1}\left[P_{p+1}\left(u / u_{0}\right)-P_{p-1}\left(u / u_{0}\right)\right]
$$

where the normalization constant

$$
g=\frac{(p+1) !}{(2 p+1) ! !}
$$


is inserted to guarantee that the highest order term in (3.24) is just $u^{p+1}$, as in (3.2). Incidentally, the coefficient in front of $u^{p-1}$ in (3.24) determines the relation between $t_{0}$ in (3.2) and the cosmological constant,

$$
t_{0}=-\frac{1}{2} \frac{p(p+1)}{2 p-1} u_{0}^{2}
$$

Eq.(3.19) yields

$$
Z_{0}=u_{0}^{2 p+3} \frac{g^{2}(2 p+1)}{(2 p+3)(2 p-1)}
$$

and then from (3.21)

$$
\frac{Z_{k k^{\prime}}}{Z_{0}}=\delta_{k, k^{\prime}} \frac{\mathcal{N}_{p} \mu^{-k-2}}{2 p-2 k-1} \operatorname{Leg}_{M}^{2}(k),
$$

where $\mathcal{N}_{p}$ is the same as in (2.17), and

$$
\operatorname{Leg}_{M}(k)=\frac{g_{k} C_{k}}{(2 p+1) g a_{0}^{k+2}} .
$$

Eq.(3.28) reproduces the structure of the two-point numbers in (2.24), and the identity of (3.29) with the leg factors (2.18) fixes the normalization constants $C_{k}$. Eq.(3.22), (3.24) are equivalent to Eq. (4.24), (4.28) in Ref. [8]7.

\section{Three- and four-point correlation numbers}

Before proceeding to the higher-order correlation numbers, it is useful to get rid of annoying factors in (3.22) and (3.24). We trade $\lambda_{k}$ for the dimensionless couplings

$$
s_{k}=\frac{g_{k} u_{0}^{-k-2}}{g(2 p+1)} \lambda_{k}
$$

${ }^{7}$ The relation

$$
\int_{1}^{\infty} P_{n}(x) e^{-l x}=\sqrt{\frac{2}{\pi l}} K_{n+1 / 2}(l)
$$

between the Legendre polynomials and the Macdonald functions of half-integer order makes the identity evident. 
and write the polynomial (3.2) as

$$
\mathcal{P}(u)=g(2 p+1) u_{0}^{p+1} Q\left(u / u_{0}\right),
$$

where $Q(x)$ is the polynomial of degree $p+1$; as in (3.11), we will think of it as the power series in $s_{k}$,

$$
Q(x)=Q_{0}(x)+\sum_{k=1}^{p-1} s_{k} Q_{k}(x)+\sum_{k_{1} k_{2}}^{p-1} \frac{s_{k_{1}} s_{k_{2}}}{2} Q_{k_{1} k_{2}}(x)+\ldots
$$

Eq's (3.24) and (3.22) then tell us that

$$
Q_{0}(x)=\frac{P_{p+1}(x)-P_{p-1}(x)}{2 p+1}=\int P_{p}(x) d x
$$

and

$$
Q_{k}(x)=P_{p-k-1}(x) .
$$

It is convenient also to trade the partition function (3.3) for the dimensionless quantity $\mathcal{Z}=\mathcal{Z}\left(s_{1}, \ldots, s_{p-1}\right)$

$$
Z=g^{2}(2 p+1)^{2} u_{0}^{2 p+3} \mathcal{Z}
$$

given by

$$
\mathcal{Z}=\frac{1}{2} \int_{1}^{x_{*}} Q^{2}(x) d x+\frac{1}{4} \int_{-1}^{1} Q^{2}(x) d x,
$$

where $x_{*}=x_{*}\left(s_{1}, \ldots, s_{p-1}\right)$ is the largest real root of $Q(x)$. Note that $x_{*}(0,0, \ldots, 0)=1$, and

$$
Q_{0}^{\prime}(1)=1, \quad Q_{k}(1)=1 .
$$

Up to the leg factors, the correlation numbers are the ratios

$$
\mu^{\sum \delta_{k_{i}}} \mathcal{Z}_{k_{1} k_{2} \ldots k_{n}} / \mathcal{Z}_{0}
$$

of the coefficients of the expansion

$$
\mathcal{Z}=\mathcal{Z}_{0}+\sum_{k=1}^{p-1} s_{k} \mathcal{Z}_{k}+\ldots+\sum_{k_{1}, \ldots k_{n}=1}^{p-1} \frac{s_{k_{1}} \ldots s_{k_{n}}}{n !} \mathcal{Z}_{k_{1} \ldots k_{n}}+\ldots
$$

Note that with (4.4), Eq.(4.7) yields

$$
\mathcal{Z}_{0}=\frac{1}{4} \int_{-1}^{1} Q_{0}^{2}(x) d x=\mathcal{N}_{p}^{-1},
$$

where $\mathcal{N}_{p}$ is precisely the factor (2.17). 


\subsection{Three point numbers}

Evaluation of the coefficients $\mathcal{Z}_{k_{1} k_{2} k_{3}}$ is straightforward; we put the calculations away into Appendix B. The result is

$$
\mathcal{Z}_{k_{1} k_{2} k_{3}}=-1+\frac{1}{2} \int_{-1}^{1}\left[Q_{k_{1} k_{2}}(x) Q_{k_{3}}(x)+Q_{k_{1} k_{3}}(x) Q_{k_{2}}(x)+Q_{k_{2} k_{3}}(x) Q_{k_{1}}(x)\right] d x,
$$

The first term -1 reproduces (2.16), except for the fusion rule factor $N_{k_{1} k_{2} k_{3}}$, Eq's (2.20), (2.21). The role of the second term is to fix that discrepancy. Note that in this case we need not worry about the odd sector. Recall from (3.10) that when $k_{1}+k_{2}+k_{3}$ is odd and $<2 p-1$, i.e. when the odd-sector fusion rules (2.20) are violated, the terms with $Z_{k_{1} k_{2} k_{3}}$ belong to the regular part of the partition function. Therefore, we only need to look at the case when $k_{1}+k_{2}+k_{3}$ is even, where (2.21) demands that the second term in (4.12) turns to 1 at all configurations of $k_{1}, k_{2}, k_{3}$ such that $k_{1}+k_{2}>k_{3}$ (as in (2.21) we assume that $k_{1}, k_{2} \leq k_{3}$ ), to cancel the first term in (4.12). To reproduce the fusion rule factor $N_{k_{1} k_{2} k_{3}}$ we need to have

$$
\frac{1}{2} \int_{-1}^{1} Q_{k_{3}}(x) Q_{k_{1} k_{2}}(x) d x=\left\{\begin{array}{lll}
1 & \text { if } & k_{1}+k_{2}<k_{3} \\
0 & \text { if } & k_{1}+k_{2} \geq k_{3}
\end{array}\right.
$$

Since $Q_{k}(x)=P_{p-k-1}(x)$, this is achieved by taking (see Eq.(A.8) in the Appendix A)

$$
Q_{k_{1} k_{2}}(x)=P_{p-k_{1}-k_{2}-2}^{\prime}(x)
$$

where prime denotes the derivative of the Legendre polynomial with respect to $x$. Note that now for some admissible values of $k_{1}, k_{2}$ the index in (4.14) can take negative values; throughout this paper we adopt the convention that $P_{n}(x)$ with negative $n$ are identically zero. With (4.14), we have

$$
\mathcal{Z}_{k_{1} k_{2} k_{3}} / \mathcal{Z}_{0}=-N_{k_{1} k_{2} k_{3}} \mathcal{N}_{p}
$$

in exact agreement with (2.16).

\subsection{Four point numbers}

Direct calculation (Appendix B) yields

$$
\mathcal{Z}_{k_{1} k_{2} k_{3} k_{4}}=\mathcal{Z}_{k_{1} k_{2} k_{3} k_{4}}^{(0)}+\mathcal{Z}_{k_{1} k_{2} k_{3} k_{4}}^{(\mathrm{I})}
$$


where

$$
\mathcal{Z}_{k_{1} k_{2} k_{3} k_{4}}^{(0)}=-F(-2)+\sum_{i=1}^{4} F\left(k_{i}-1\right)-F\left(k_{(12 \mid 34)}\right)-F\left(k_{(13 \mid 24)}\right)-F\left(k_{(14 \mid 23)}\right),
$$

and

$$
\mathcal{Z}_{k_{1} k_{2} k_{3} k_{4}}^{(\mathrm{I})}=\frac{1}{2} \int_{-1}^{1}\left[Q_{k_{1} k_{2} k_{3}} Q_{k_{4}}+Q_{k_{4} k_{1} k_{2}} Q_{k_{3}}+Q_{k_{3} k_{4} k_{1}} Q_{k_{2}}+Q_{k_{2} k_{3} k_{4}} Q_{k_{1}}\right] d x .
$$

$\operatorname{In}(4.17)$

$$
F(k)=P_{p-k-2}^{\prime}(1)=\frac{1}{2}(p-k-1)(p-k-2) \Theta_{p-2, k},
$$

with $\Theta_{k, k^{\prime}}$ being the step function

$$
\Theta_{k, k^{\prime}}= \begin{cases}1 & \text { for } \quad k \geq k^{\prime} \\ 0 & \text { for } \quad k<k^{\prime}\end{cases}
$$

and we use the notation

$$
k_{(i j \mid l m)}=\min \left(k_{i}+k_{j}, k_{l}+k_{m}\right) .
$$

Like in (4.12), the role of the term (4.18) is to enforce the fusion rules, and the polynomials $Q_{k_{1} k_{2} k_{3}}(x)$ are to be determined from this requirement.

The analysis is more simple in the even sector, so let's start with this case. Assume again that the numbers $k_{1}, k_{2}, k_{3}, k_{4}$ are arranged as in (2.27), so that in (4.17) we always have

$$
k_{(12 \mid 34)}=k_{1}+k_{2}, \quad k_{(13 \mid 24)}=k_{1}+k_{3} .
$$

Recalling (4.5), and counting the degrees of the polynomials $Q_{k_{1} k_{2} k_{3}}(x)$, one observes that (4.18) vanishes when the even sector fusion rules (2.7) are satisfied. On the other hand, when the fusion rules are violated, the inequality (2.28) is violated as well, and we have

$$
k_{(14 \mid 23)}=k_{2}+k_{3}<p-1
$$

where the last inequality follows from $k_{4} \leq p-1$. With (4.22) and (4.23) the expression (4.17) evaluates to

$$
-\frac{1}{2}\left(k_{4}-k_{1}-k_{2}-k_{3}-2\right)\left(2 p-3-k_{1}-k_{2}-k_{3}-k_{4}\right) .
$$


Thus, for (4.16) to satisfy the even-sector fusion rules (2.7) the integral

$$
\int_{-1}^{1} Q_{k_{1} k_{2} k_{3}}(x) Q_{k_{4}}(x) d x
$$

has to return (4.24) with the opposite sign. This uniquely determines the polynomials $Q_{k_{1} k_{2} k_{3}}$

$$
Q_{k_{1} k_{2} k_{3}}(x)=P_{p-\sum k_{i}-3}^{\prime \prime}(x)
$$

(see Eq.(A.9) in Appendix A).

Now, in the odd sector, as was explained in Sect.2.2, the coefficients (4.16) with $\sum k_{i} \leq$ $2 p-5$ correspond to regular terms in the partition function $Z$, and thus can be disregarded. For that reason, in particular, we can pay no attention to the second term, Eq.(4.18). The only singular terms potentially violating the odd-sector fusion rules (2.7) are those with

$$
\sum k_{i}=2 p-3
$$

But it is easy to check that these terms actually vanish. Indeed, if again $k_{i}$ are arranged as in (2.27), and $k_{1}+k_{4} \leq k_{2}+k_{3}$ (in which case it follows from (4.27) that also $k_{1}+k_{4} \leq p-1$ ), the (4.17) is given by (4.24) which vanishes at (4.27). If instead $k_{2}+k_{3} \leq k_{1}+k_{4}$ (and hence $k_{2}+k_{3} \leq p-1$ ), the expression (4.17) reduces to

$$
\left(k_{1}+1\right)\left(2 p-3-k_{1}-k_{2}-k_{3}-k_{4}\right),
$$

again vanishing at (4.27).

Once the fusion rules are taken care of, we can compare (4.16) with the $\mathcal{M G}_{2 / 2 p+1}$ fourpoint correlation number. It is not difficult to check that when (2.28) is satisfied, (4.17) reproduces (2.26) perfectly.

However, when instead $k_{2}+k_{3}<k_{1}+k_{4}$, (4.17) no longer agrees with (2.26). This is expected, since the Liouville Gravity calculations in [6] were made under a certain assumption which is violated in this case. The Matrix Model result (4.16), which is valid at all configurations of $k_{i}$, may provide a hint on how to modify the Liouville Gravity analysis when the number of conformal blocks in the $\mathcal{M}_{2 / 2 p+1}$ four-point function is smaller then $k_{1}$.

\section{Multi-Point correlation numbers}

In principle, one can extend the analysis of the previous Section to $n$-point correlation numbers with $n>4$. Although at the moment no specific results for these multi-point 
numbers in the Minimal Gravity are available, one thing is known. Since the correlation functions (2.4) of $\mathcal{M}_{2 / 2 p+1}$ vanish when the fusion rules (2.7) are violated, the correlation numbers (2.14) then vanish as well. This requirement for the $n$-point numbers imposes strong conditions on the form of the polynomials $Q_{k_{1} \ldots k_{n-1}}(x)$, which fix them uniquely, and thus one must be able to determine the complete form of the polynomial $Q(x)$, Eq.(4.3), step by step in $n$.

In fact, the problem seems over-determined. Indeed, suppose we have already constructed the expansion (4.3) up to the order $n-1$, and thus $Q_{0}, Q_{k}, \ldots, Q_{k_{1} \ldots k_{n-2}}$ are already determined. Then $Q_{k_{1} \ldots k_{n-1}}$ enters the expression for the $n$-th order coefficient $\mathcal{Z}_{k_{1} \ldots k_{n}}$ only through the "counterterm"

$$
\frac{1}{2} \int_{-1}^{1} Q_{k_{1} \ldots k_{n-1}}(x) Q_{k_{n}}(x) d x
$$

and $n$ similar terms which differ from this by cyclic permutations of $k_{1}, \ldots, k_{n}$. The polynomials $Q_{k_{1} \ldots k_{n-1}}$ must be chosen in such a way that these terms cancel all other contributions to $\mathcal{Z}_{k_{1} \ldots k_{n}}$ when the even-sector fusion rules (2.7) are violated, i.e. when $k_{1}+\ldots+k_{n-1}>k_{n}$. But since the degree of the polynomial $Q_{k_{1} \ldots k_{n-1}}(x)$ is $p+3-2 n-\left(k_{1}+\ldots+k_{n-1}\right)$ (see Eq.(3.13)), the integral (5.1) actually vanishes at $k_{1}+\ldots+k_{n-1}>k_{n}+4-2 n$. For $n \geq 4$ a window

$$
k_{n}>\sum_{i=1}^{n-1} k_{i}>k_{n}+4-2 n
$$

opens in configurations of $k_{i}$ violating the even-sector fusion rules, where the term (5.1) seems to be incapable of doing its job of fixing the fusion rules. A similar problem exists in the odd sector. For $n \geq 4$ there is a window

$$
2 p-1>\sum_{i=1}^{n} k_{i}>2 p+3-2 n
$$

in the configurations of $k_{i}$, where the odd-sector fusion rules (2.7) are violated, but corresponding coefficients $Z_{k_{1} \ldots k_{n}}$ are singular (involve negative integer powers of $\mu$, see Sect. $3.1)$.

We have seen in Section 4.2 that at $n=4$ the problem takes care of itself, in both even and odd sectors. The first factor in Eq.(4.24) forces the coefficients $Z_{k_{1} k_{2} k_{3} k_{4}}$ to vanish within the window (5.2), and the last factor in (4.24) and (4.28) makes sure that they vanish within the window (5.3) as well. 
The same phenomenon should persist for higher $n>4$, for otherwise we would face incurable disagreement between the correlation numbers in $\mathcal{M G}_{2 / 2 p+1}$ and the Matrix Model. We have calculated the five-point correlation numbers $C_{k_{1} k_{2} k_{3} k_{4} k_{5}}$, and indeed they automatically vanish within both even and odd sector windows, Eq.(5.2) and Eq.(5.3). We do not present this calculation here, for the result is somewhat cumbersome, and anyway at the moment there is nothing on the Minimal Gravity side to compare it with. But as the byproduct of this calculation we have determined the four-index polynomials $Q_{k_{1} \ldots k_{4}}$,

$$
Q_{k_{1} k_{2} k_{3} k_{4}}(x)=P_{p-\sum k-4}^{\prime \prime \prime}(x),
$$

where $\sum k=k_{1}+k_{2}+k_{3}+k_{4}$.

\section{Discussion}

Identification of $\mathcal{M G}_{2 / 2 p+1}$ as the world-sheet theory of the $p$-critical one-Matrix Model suggests that, by choosing suitable resonance terms in the of the relation between the couplings $t_{k}$ in (3.2) and $\lambda_{k}$, the Matrix Model correlation numbers can be put in agreement with the fusion rules of $\mathcal{M G}_{2 / 2 p+1}$. Technically, this is done by constructing the polynomial $Q(x)$, Eq.(4.3), order by order in $s_{k}$. In Sections 4 and 5 above, we have executed this program up to the fourth order.

For higher $n$ direct calculations become rather involved. But a quick glance at (4.5), (4.14), (4.26), and (5.4) immediately suggests the general form,

$$
Q_{k_{1} \ldots k_{n}}(x)=\left(\frac{d}{d x}\right)^{n-1} P_{p-\sum k-n}(x),
$$

where again $\sum k=k_{1}+\ldots+k_{n}$. Then, using (A.6), the full polynomial $Q(x)$ can be neatly written as the integral

$$
Q(x)=-\oint_{0} \frac{\left(1-2 x z+z^{2}-\sum_{k=1}^{p-1} 2 s_{k} z^{k+2}\right)^{1 / 2}}{z^{p+2}} \frac{d z}{2 \pi i},
$$

where the integration is over a small circle around 0 . So far, we did not find a proof that all correlation numbers computed from (4.7) with $Q(x)$ given by (6.2) obey all fusion rules (2.7). Thus, (6.2) remains a conjecture. 


\section{Appendix}

\section{A Legendre Polynomials}

The Legendre polynomials $P_{n}(x)$ are $n$-th order polynomials which form an orthogonal system on the interval $[-1,1]$ with the weight 1 ,

$$
\int_{-1}^{1} P_{n}(x) P_{n^{\prime}}(x) d x=\frac{2 \delta_{n, n^{\prime}}}{2 n+1} .
$$

The standard normalization is such that

$$
P_{n}(1)=1
$$

Explicitly,

$$
P_{n}(x)=\frac{2^{-n}}{n !} \frac{d^{n}}{d x^{n}}\left[x^{2}-1\right]^{n}=2^{-n} \sum_{l=0}^{[n / 2]}(-)^{l} \frac{(2 n-2 l) !}{l !(n-l) !(n-2 l) !} x^{n-2 l} .
$$

Another closed expression is given in terms of the Hypergeometric series,

$$
P_{n}(x)={ }_{2} F_{1}\left(-n, n+1,1 ; \frac{1-x}{2}\right) \text {, }
$$

i.e.

$$
P_{n}^{\prime}(1)=\frac{n(n+1)}{2}, \quad P_{n}^{\prime \prime}(1)=\frac{(n-1) n(n+1)(n+2)}{8}, \quad \text { etc }
$$

Yet another closed form is in terms of the contour integral

$$
P_{n}(x)=\oint_{0} \frac{\left(1-2 x z+z^{2}\right)^{-1 / 2}}{z^{n+1}} \frac{d z}{2 \pi i}
$$

The following relations are useful in our analysis:

$$
P_{n+1}^{\prime}(x)-P_{n-1}^{\prime}(x)=(2 n+1) P_{n}(x) ;
$$

they are valid for all $n=0,1,2,3, \ldots$ if one assumes that $P_{-1}(x)=0$. 
Besides the orthogonality condition (A.1), we need integrals involving the derivatives of the Legendre polynomials,

$$
\begin{gathered}
\frac{1}{2} \int_{-1}^{1} P_{n}^{\prime}(x) P_{m}(x) d x=E_{n+m-1} \Theta_{n, m+1} \\
\frac{1}{2} \int_{-1}^{1} P_{n}^{\prime \prime}(x) P_{m}(x) d x=E_{n+m} \Theta_{n, m+2} \frac{(n+m+1)(n-m)}{2},
\end{gathered}
$$

and in general

$$
\begin{aligned}
\frac{1}{2} \int_{-1}^{1} P_{n}^{(l)}(x) P_{m}(x) d x=E_{n+m-l} \Theta_{n, m+l} \times \\
\quad \frac{2^{-l+1}}{(l-1) !} \prod_{s=0}^{l-2}(n+m+l-1-2 s)(n-m+l-2-2 s),
\end{aligned}
$$

where $P^{(l)}(x)$ stands for the $l$-th derivative. Here $\Theta_{n, m}=P_{n-m}(1)$ is the step function (4.20), and

$$
E_{n}=\left\{\begin{array}{llll}
1 & \text { if } & n & \text { is even } \\
0 & \text { if } & n & \text { is odd }
\end{array} .\right.
$$

Integrating (A.9) by parts, we have

$$
\frac{1}{2} \int_{-1}^{1} P_{n}^{\prime}(x) P_{m}^{\prime}(x) d x=E_{n+m}\left[\Theta_{m, n} \frac{n(n+1)}{2}+\Theta_{n, m} \frac{m(m+1)}{2}\right] .
$$

\section{B Evaluation of $\mathcal{Z}_{k_{1} \ldots k_{n}}$}

Generally, the coefficient $\mathcal{Z}_{k_{1} \ldots k_{n}}$ is computed by taking $n$-th order derivative of (4.7) with respect to the parameters $s_{k_{1}}, \ldots, s_{k_{n}}$. The result naturally splits into two pieces

$$
\mathcal{Z}_{k_{1} \ldots k_{n}}=\mathcal{Z}_{k_{1} \ldots k_{n}}^{(*)}+\mathcal{Z}_{k_{1} \ldots k_{n}}^{(\mathrm{int})}
$$

corresponding to two terms (4.7). Since $x_{*}=1+O\left(s_{k}\right)$, the term $\mathcal{Z}_{k_{1} \ldots k_{n}}^{(*)}$ appears only starting from $n=3$, and in general it has "local" form, i.e. it is built from the polynomials $Q_{0}(x), Q_{k}(x), \ldots$ and their $x$-derivatives, taken at $x=1$. On the other hand, the term 
$\mathcal{Z}_{k_{1} \ldots k_{n}}^{(\mathrm{int})}$ represents explicit dependence of the integrand in the second term in (4.7) on the parameters $s_{k}$; starting from $n=3$, it exists entirely due to the presence of non-linear terms in the transformation (3.5). Generally, it has the form

$$
\mathcal{Z}_{k_{1} \ldots k_{n}}^{(\mathrm{int})}=\sum_{r=1}^{\left[\frac{n-1}{2}\right]} \frac{1}{2} \int_{-1}^{1}\left[Q_{l_{1} \ldots l_{r}}(x) Q_{l_{r+1} \ldots l_{n}}(x)\right] d x
$$

where $\left(l_{1}, \ldots, l_{n}\right)$ are permutations of the numbers $\left(k_{1}, \ldots, k_{n}\right)$, and the symbol $[\ldots]$ signifies the sum of all distinct terms generated by the permutations.

Calculation of the "local" term $\mathcal{Z}_{k_{1} \ldots k_{n}}^{(*)}$ is more tedious since it involves taking derivatives of $x_{*}$, but it can be streamlined by reducing it to the residue calculus. Write

$$
\int_{1}^{x_{*}} Q^{2}(x) d x=\oint_{1, x_{*}} Q^{2}(x) \log \frac{Q(x)}{Q_{0}(x)} \frac{d x}{2 \pi i} .
$$

where the integration contour encircles the points 1 and $x_{*}$, but leaves outside all other roots of $Q(x)$ and $Q_{0}(x)$. For the sake of the expansion in $s_{k}$, we may write $Q(x)$ as

$$
Q(x)=Q_{0}(x)+\tilde{Q}(x),
$$

where $\tilde{Q}(x)$ represents all the $s_{k}$-dependent terms in the expansion (4.3), so that (B.3) takes the form

$$
\frac{1}{2} \oint_{1, x_{*}} Q_{0}^{2}(x) R\left(\tilde{Q}(x) / Q_{0}(x)\right) \frac{d x}{2 \pi i}
$$

with $R(t)=(1+t)^{2} \log (1+t)$. When $R(t)$ is expanded as the power series

$$
R(t)=t+\frac{3}{2} t^{2}+\frac{1}{3} t^{3}-\frac{1}{12} t^{4}+\frac{1}{30} t^{5}+\ldots
$$

the $l$-th term produces $l$-th order pole at $x=1$ in the integrand in (B.5). Then the local terms are computed by evaluating the residues.

\section{Results}

For $n=3$ one finds

$$
\mathcal{Z}_{k_{1} k_{2} k_{3}}=-\frac{Q_{k_{1}}(1) Q_{k_{2}}(1) Q_{k_{3}}(1)}{Q_{0}^{\prime}(1)}+\frac{1}{2} \int_{-1}^{1}\left[Q_{l_{1} l_{2}}(x) Q_{l_{3}}(x)\right] d x,
$$


which reduces to (4.12) in view of (4.8).

For $n=4$ we have

$$
\mathcal{Z}_{k_{1} k_{2} k_{3} k_{4}}^{(*)}=-\frac{\left[Q_{l_{1}} Q_{l_{2}} Q_{l_{3}} Q_{l_{4}}\right] Q^{\prime \prime}}{\left(Q_{0}^{\prime}\right)^{3}}+\frac{\left[Q_{l_{1}}^{\prime} Q_{l_{2}} Q_{l_{3}} Q_{l_{4}}\right]}{\left(Q_{0}^{\prime}\right)^{2}}-\frac{\left[Q_{l_{1} l_{2}} Q_{l_{3}} Q_{l_{4}}\right]}{Q_{0}^{\prime}}
$$

where all the $Q$ 's are taken at $x=1$, and the brackets [...] have the same meaning as in (B.2), for instance the last term is in fact the sum of six terms.

The integral term in this case has two parts,

$$
\mathcal{Z}_{k_{1} k_{2} k_{3} k_{4}}^{(\mathrm{int})}=\frac{1}{2} \int_{-1}^{1}\left[Q_{l_{1} l_{2}}(x) Q_{l_{3} l_{4}}(x)\right]+\frac{1}{2} \int\left[Q_{l_{1} l_{2} l_{3}}(x) Q_{l_{4}}(x)\right] d x
$$

The first term here involves only the polynomials $Q_{k k^{\prime}}(x)$ given by (4.14); it is evaluated using (A.13), and when combined with (B.8), yields (4.17). The second part in (B.9) provides (4.18).

\section{Acknowledgments}

We are grateful to P.Di Francesco, V.Kazakov, I.Krichever, A.Marshakov, G.Moore, A.Polyakov, and M.Staudacher for interest to this work and discussions. AZ would like to acknowledge warm hospitality he received at the Bogoliubov Lab at JINR, Dubna, in the spring of 2008, when this work was done.

AB is supported by the grants RFBR 07-02-00799, and SS-3472.2008.2. The work of AZ is supported by DOE under grant DE-FG02-96ER40949.

\section{References}

[1] A.Polyakov, "Quantum Geometry of Bosonic Strings", Phys.Lett.B103:207-210,(1981).

[2] P.H.Ginsparg and G.W.Moore, "Lectures on 2-D gravity and 2-D string theory", arXiv:hep-th/9304011;

P.Di Francesco, P.H.Ginsparg, J.Zinn-Justin, "2-D Gravity and random matrices", Phys.Rep.254:1-133,(1995), hep-th/9306153

[3] V.G.Knizhnik, A.M.Polyakov, A.B.Zamolodchikov, "Fractal Structure of 2D Quantum Gravity", Mod.Phys.Lett.A3:819,(1988) 
[4] M.Goulian, M.Li, "Correlation Functions in Liouville Theory", Phys.Rew. Letters, 66, 2051:2055 (1991)

[5] P.Di Francesco, D.Kutasov, "World Sheet and Space Time Physics in Two Dimensional (Super) String Theory", Nucl.Phys. B375 (1992) 119-172

[6] A.Belavin, Al.Zamolodchikov, "Moduli integrals, ground ring and four-point function in minimal Liouville gravity", Theor.Math.Phys. 147:729-754,(2006); hep-th/0510214, pages $16-46$.

[7] Al.Zamolodchikov, "Higher equations of motion in Liouville field theory", Int.J.Mod.Phys. A19S2:510-523,(2004), hep-th/0312279

[8] G.W.Moore, N.Seiberg, M.Staudacher, "From loops to states in 2-D quantum gravity", Nucl. Phys. B362,665-709, (1991)

[9] Al.Zamolodchikov, "Three-point function in the minimal Liouville gravity", Theor.Math. Phys.142:183-196,(2005)

[10] M.Staudacher, "The Yang-Lee Edge Singularity On A Dynamical Planar Random Surface", Nucl. Phys.B336:349,1990.

[11] F.David, "Conformal Field Theories Coupled to 2D Gravity in the Conformal Gauge", Mod.Phys.Lett.A3:1651,1988;

J.Distler, H.Kawai, "Conformal Field Theory and 2D Quantum Gravity Or Who's Afraid of Joseph Liouville?" Nucl.Phys.B321:509,(1989)

[12] J.Cardy, "Conformal Invariance And The Yang-Lee Edge Singularity In Two Dimensions", Phys.Rev.Lett.54:1354-1356,(1985)

[13] E.Witten, "On The Structure of The Topological Phase of Two-Dimensional Gravity", Nucl. Phys. B340:281-332, (1990)

[14] H.Bateman, A.Erdélyi, "Higher Transcendental Functions", V.2, Mc Graw-Hill, (1953) 